\title{
De la fragmentation procédurale à l'émiettement épistémologique
}

Quelles articulations pour la production discursive?

Bertrand Labasse, Département de communication et Département de français, Université d'Ottawa,

K1N 6N5 Ottawa (Ontario)

blabasse.udo@pressetech.org 


\title{
Résumé
}

Le développement social, économique et technologique du domaine de la communication s'accompagne d'une spécialisation croissante des pratiques professionnelles et des formations qui leurs sont liées, au risque d'un délitement de la substance académique de ces dernières. Un tel délitement est-il pour autant inéluctable ? Se consacrant au dénominateur commun de ces filières - la production discursive médiatisée sous toutes ses formes - cet article examinera l'hypothèse selon laquelle les problèmes de cohérence perceptibles à l'échelle globale, celle de l'interdiscipline, sont structurellement assimilables à ceux que peut connaître, à l'échelle locale, tout programme de formation universitaire en communication, lesquels sont à leur tour similaires à ceux que l'on peut remarquer à l'échelle d'un simple cours ou même d'un manuel de communication à vocation pratique. On examinera dans un premier temps - en prolongeant une recherche antérieure comment les connaissances et préceptes opératoires sont structurés dans les manuels, avant de transposer cette analyse à la structuration interne des programmes de formation, ce qui conduira enfin à montrer que la cohérence et les perspectives de la nébuleuse interdisciplinaire que forme l'enseignement de la communication dépend moins des bourgeonnements de sa périphérie que des articulations fondamentales qui en établissent le centre.

Mots clés : communication, épistémologie, didactique, expertise, rédactologie

\begin{abstract}
Social, economic and technological developments in the field of communication are accompanied by increasing specialization of professional activities and academic programs, to the risk of a disintegration of the academic substance of the field. Is such a disintegration inevitable? Focusing on the common denominator of these curricula - discursive production in all its forms - this article will examine the hypothesis that the problems of consistency visible globally, those of the interdiscipline, are structurally similar to those that encounter, locally, most university programs in communication, which are in turn similar to those that can be observed on the scale of a single course or even of a practical communication guide. We will first examine how the knowledge and precepts are structured in textbooks, before transposing this analysis to the internal structure of training programs, which will lead us to show that consistency and prospects for communication in higher education depends less on its burgeoning periphery that on the fundamental articulations at the center of the interdiscipline.
\end{abstract}

Keywords: communication, epistemology, didactics, expertise, writing studies 
Le champ de la communication est confronté à une évolution paradoxale. En tant que domaine de recherche, il a vu l'importance sociale et économique de son objet d'étude croître au point de devenir l'une des désignations de son époque : la société dite « de la communication ». En tant que matière d'enseignement, il bénéficie d'un engouement qui ne se dément pas : le nombre d'étudiants dans cette interdiscipline aurait, par exemple, crû en France de 58\% en dix ans (Lutinier, Dietsch et Sotto, 2011). Pourtant, ces indications en apparence très favorables pourraient aussi être le signe d'une sévère crise de croissance. Le développement social et économique de la communication s'accompagne d'un éclatement des technologies et des pratiques semblant accroître la fragmentation d'une interdiscipline dont la cohérence soulevait déjà des inquiétudes il y a presque 40 ans (Escarpit, 1976). De même, la multiplication des formations en communication privées ou publiques, y compris au sein d'autres disciplines paraît tendre à diluer l'assise même de ce champ (Lépine et De la Broise, 2012), tandis que ses propres étudiants se trouvent en concurrence avec d'autres sur le marché de l'emploi (Sacré, 1998). Ainsi, le problème de sa spécificité (Olsen, Weber et Trimble, 2002), de sa pertinence et, plus généralement, de l'expertise qu'il confère à ses diplômés se présente-t-il de façon fort délicate : vouloir renforcer sa valeur instrumentale en se rapprochant d'attentes et de pratiques professionnelles morcelées conduirait-il nécessairement à déliter sa substance ?

Cet article se propose de montrer qu'une telle contradiction, qui paraît se poser à une échelle globale - celle de la composition de chaque programme universitaire (la macrostructure) - peut pourtant être discernée dans la structuration interne des discours pédagogiques (la microstructure) en particulier lorsque la matière qui est enseignée est résolument «pratique». Or, si la microstructuration des contenus procéduraux s'avère reproduire à petite échelle certains des problèmes qui affectent leur macrostructuration, examiner la première peut aider à éclairer la seconde, et, à partir de là, à examiner des voies pour dépasser la contradiction évoquée plus haut.

\section{Les préceptes opératoires en communication écrite}

Si l'enseignement de la communication couvre un spectre toujours plus large de pratiques professionnelles, il n'en paraît pas moins présenter une cohérence réelle dès lors que l'on considère ce que ces spécialités peuvent avoir de commun. Sous une forme ou sous une autre, elles se rapportent en effet fondamentalement à la diffusion de représentations au moyen de discours adaptés aux publics visés. Les variables - formes et médias, contexte organisationnel, objectifs et contraintes, publics... - peuvent évoluer, mais la capacité à produire des discours appropriés demeure le centre de gravité de cet ensemble et son principal objectif opératoire, de même que l'étude de ces discours - de leur production à leur réception - est ce qui le 
constitue en théorie comme "a coherent field (...) of discourse about discourse " (Craig, 1999, p. 120).

Il va sans dire que la production discursive n'est pas une compétence «nue»: qu'il s'agisse de concevoir des pages web, des communiqués de presse ou des scripts vidéo, elle suppose le plus souvent des connaissances approfondies, tant techniques (par exemple JavaScript) que contextuelles (par exemple la sociologie des organisations ou des médias) et référentielles (par exemple en vulgarisation scientifique), mais la communication n'en demeure pas moins, dans son essence, un « ars bene dicendi ».

Dans ce cadre, l'aptitude à construire des discours textuels "appropriés » (efficaces, clairs, attractifs, etc.) apparaît comme quadruplement digne d'intérêt ;

- d'une part parce qu'elle se trouve non seulement au cœur de la communication écrite en tant que telle, mais aussi en amont de bien des formes de communication orale ou audio visuelle ${ }^{44}$;

- d'autre part parce qu'elle constitue, en pratique, un important critère d'appréciation des diplômés (et un motif courant d'insatisfaction des employeurs);

- ensuite parce qu'elle constitue un élément de différentiation majeur entre le champ de la communication et les autres disciplines, tant techniques (technologies de l'information) qu'artistiques (graphisme) revendiquant des débouchés professionnels voisins, en particulier dans la sphère d'Internet ;

- enfin parce qu'elle intègre - de l'inventio à l'elocutio - l'essentiel des compétences fondamentales en communication: comprendre une mission, rassembler et évaluer sa documentation, organiser ses idées, percevoir les contraintes, anticiper la réception, etc.

Pour autant, les travaux portant sur les connaissances mobilisées dans le domaine de la communication écrite professionnelle mais aussi sur le processus de transposition didactique de celles-ci ou, plus prosaïquement, sur l'expertise qu'elles sont susceptibles de conférer, demeurent extrêmement rares. Il est vrai que l'observation des situations d'enseignement réelles permettrait difficilement de constituer un inventaire large, détaillé et généralisable, celles-ci ne pouvant se référer à un corpus de « savoirs à enseigner » consensuel (Chevallard, 1985) et étant souvent déléguées à des chargés de cours.

C'est ce qui a conduit à la constitution d'une base de référence, obtenue par le dépouillement approfondi et la mise en concordance thème par thème de cinquante manuels de rédaction publiés dans trois spécialités, le journalisme, la communication d'entreprise et la rédaction technique, scientifique et médicale

\footnotetext{
${ }^{44}$ Par exemple la logographie, les scripts et scénarios ou encore les guides d'entretien en marketing téléphonique, pour ne pas parler de tous les écrits de prospection (propositions) et d'administration qu'impliquent l'activité professionnelle des entreprises de communication.
} 
$(\text { Labasse, 2006) })^{45}$. Ce travail se fondait sur l'hypothèse que la majorité de ces ouvrages étaient issus de l'expérience pédagogique de leurs auteurs ${ }^{46}$, mais aussi sur l'hypothèse qu'il était hautement improbable que des connaissances, préceptes ou recettes d'une certaine importance ou représentativité aient pu échapper simultanément à tous ces ouvrages.

La plupart des enseignements issus de cette analyse ayant déjà été détaillés ailleurs, on se contentera ici de les résumer un peu abruptement.

- Les ouvrages manifestent souvent une stratégie de distinction assez marquée, tant verticale (par rapport à la rédaction scolaire) qu'horizontale (par rapport aux autres domaines d'application).

- La distinction horizontale, revendiquée surtout dans les cas du journalisme et de la rédaction technique, ne permet pas pour autant aux auteurs de définir l'essence et les contours de leur objet: les démarcations proposées, quand elles ne sont pas discutables dans les faits, pourraient s'appliquer à de nombreux autres types de textes (il est significatif que les dix manuels consacrés à la rédaction technique ${ }^{47}$, s'ils présentent de larges similitudes de contenu avec les autres livres du corpus, ne s'accordent en revanche pas du tout entre eux sur les genres d'écrits qu'ils couvrent ${ }^{48}$ ) ;

\footnotetext{
${ }^{45}$ Ces manuels, publiés en deux langues dans six pays francophones ou anglophones ont été selectionnés par une méthode semi-aléatoire : certains ont été choisis pour leur notoriété (Flesch, Richaudeau...) et d'autres en fonction de quotas entre les sous-champs (en matière de communication scientifique et de journalisme, on s'est efforcé d'inclure la totalité des ouvrages francophones disponibles au moment de l'étude) mais plus du tiers des ouvrages ont été sélectionnés au hasard sur la base de leur titre, le contrôle se limitant à appliquer des critères d'exclusion thématiques prédéfinis (étaient ainsi rejetés les ouvrages portant sur l'écriture scolaire ou de fiction, la correspondance commerciale ou privée, etc.). Au total, le corpus englobait l'essentiel de la production francophone et un échantillon qualitativement représentatif de la production anglophone. On en trouvera la liste complète dans Labasse (2006) et Labasse (2009), accompagnée de précisions méthodologiques trop longues pour être reproduites ici. Signalons simplement que son dépouillement a recouru à plusieurs méthodes quantitatives et qualitatives, et en particulier, dans ce dernier cas, à un procédé spécifique - l'analyse de concordance topique - visant à assurer la reproductibilité des sélections textuelles (c'est-à-dire qu'un autre observateur appliquant la même méthode serait normalement conduit à retenir les mêmes assertions ou extraits).

${ }^{46}$ De fait, plus des deux tiers des ouvrages comportant des notices biographiques exploitables mentionnent un lien réel avec des formations présentielles dans l'enseignement supérieur (et, en parallèle, plus des trois quarts excipent d'une expérience professionnelle de terrain). On notera par ailleurs que 21 ouvrages comportent des exercices d'application et que 8 se destinent explicitement (à titre quasiment principal dans un cas, accessoire dans les autres) aux formateurs eux-mêmes, ce qui leur confère un intérêt programmatique particulier (ils sont à la fois « savoirs enseignés » et «savoirs à enseigner » au sens de Chevallard, op. cit.).

${ }^{47}$ Pour abréger, on désignera dorénavant ici par rédaction « technique » l'agrégat concernant la rédaction « technique, scientifique et médicale ».

48 Paradoxalement, parmi les 17 formes discursives abordées, les plus souvent évoquées sont... les schémas et graphiques (8 ouvrages sur 10). En revanche, les articles scientifiques primaires ne sont
} 
- La distinction verticale peut s'analyser comme la marque d'un basculement de priorités entre un centrage sur correction langagière (écriture scolaire) et un centrage sur l'adéquation au destinataire (écriture communicationnelle), le principe d" "écrire pour son lecteur » étant le principal point de convergence de ces ouvrages ;

- Sur le fond, les connaissances déclaratives sont peu nombreuses (et souvent erronées, comme l'évaluation à 1500 ou 3000 mots du vocabulaire du « lecteur moyen »). Pour leur part, les recommandations pratiques sont généralement avancées avec assurance, voire autorité, mais rarement expliquées. Quelques auteurs, toutefois, s'efforcent de justifier leurs conseils, mais le plus souvent en recourant à des concepts discutables, soit dénaturés (la latéralisation cérébrale de Sperry) soit pseudo-scientifiques (P.N.L., A.T.) ;

- Enfin, bien que la majorité des livres soient associés à des cours donnés dans l'enseignement supérieur, ils ne tirent aucun parti visible des six dernières décennies de recherche en sciences de la communication. Les très rares travaux scientifiques évoqués, toujours brièvement, sont essentiellement issus de la linguistique (Jakobson) et des sciences de l'information (Shannon). Encore ceux-ci paraissent-ils plus sollicités pour la caution symbolique qu'ils fournissent que pour l'intelligibilité opératoire qu'ils apportent, laquelle est difficilement discernable dans les ouvrages.

En somme, à moins que l'expertise en communication écrite ne se limite à un petit nombre de préceptes consensuels ("Dites l'important d'abord》, "Préférez les mots et les phrases courtes », etc.) et à la descriptions de normes génériques rigides, souvent assez différentes des usages professionnels réels, le bagage accumulé depuis Quintilien par les professionnels de la communication et par ceux qui les forment semble assez mince.

Il conviendrait de nuancer, en soulignant, d'une part que les remarques qui précèdent, globales et condensées, ne permettent pas de rendre justice aux efforts conceptuels de quelques auteurs et, d'autre part que, si le corpus est représentatif de la période pré-internet, il ne prend pas en compte quelques ouvrages plus récents et plus riches, axés notamment sur le "Document design $»^{49}$. Toutefois, la question n'est pas ici de soupeser à nouveau (cf. Labasse 2009) la valeur épistémologique et l'efficacité pratique des discours pédagogiques dans le domaine de la communication écrite. Il est, plus spécifiquement, d'examiner comment ces discours sont structurés.

abordés que par cinq ouvrages, les rapports techniques par trois, les modes d'emplois par trois également, etc.

${ }^{49}$ Une extension de la base de référence pour prendre en compte ces nouveaux discours est actuellement envisagée 


\section{La structuration des discours pédagogiques}

Tout manuel de communication écrite - et tout cours dans cette matière - est confronté à deux questions fondamentales: "Que peut-on bien dire de fondé et d'utile, une fois que l'on a dit "écrivez pour vos lecteurs" ? " et "Comment le dire ?». Si la première, comme on l'a vu, est délicate, la seconde ne l'est pas moins. En effet, la production d'un discours de communication de qualité professionnelle est un processus complexe, qui met en jeu de très nombreux facteurs, de nature et d'échelle très différentes et pourtant liés entre eux.

La question de savoir comment - et dans quel ordre - les ouvrages structurent ce qu'ils entendent transmettre confronte, de prime abord, à une très grande variété d'approches. Ainsi, les formes utilisées vont-elles d'un discours linéaire, revendiqué comme tel - "Il est conçu pour être lu du début à la fin. Je suppose qu'un lecteur pourrait arracher ici et là des éléments utiles, en jouant à la marelle à travers certains chapitres [...] Mais je ne le recommande pas. " (BLU88J, p. xi) - à une simple juxtaposition de fiches thématiques, plus destinées à être consultées que lues : "Chaque double page correspond à un chapitre autonome " (LAY97G p. 3). Entre ces deux extrêmes, de nombreuses stratégies sont possibles. Ainsi GRI92G tente-t-il de concilier les deux options « Guide à double entrée, ce recueil peut se parcourir au fil des pages ou point par point selon les renvois de l'index » (p. 10), tandis que FLO84J propose une succession de séances progressives au sein d'un parcours global : "l'auteur serait flatté, mais désolé, que le lecteur dévore ce livre. On en tirera un meilleur parti en l'utilisant comme un manuel : après chaque leçon, interrompre la lecture pour un exercice pratique, rédiger un article en fonction de la règle fraîchement assimilée. » (p. 7).

Malgré cette apparente diversité, il est possible de tenter de distinguer des structures types, dont le nombre s'avère, somme toute, assez réduit. Dans les faits, ces structures se combinent généralement entre elles (l'une peut-être une partie ou sous-partie d'une autre) pour former l'ossature ${ }^{50}$ de la majorité des textes dont nous disposons.

Un premier type de structure est organisé en fonction des formes textuelles de référence (genres). Bien que ce type d'agencement porte sur des réifications très différentes d'un domaine d'application à l'autre (journalisme, rédaction technique, communication d'entreprise), il est strictement identique dans son principe.

\footnotetext{
${ }^{50}$ L'ossature de la partie qu'ils consacrent à la rédaction (le reste, lorsqu'il y en a, dépasse notre propos).
} 
Une autre structuration possible, que l'on pourrait appeler procédurale (ou chronologique), tente de respecter les étapes de la production du texte, parfois depuis la recherche des informations (documentation scientifique, enquête journalistique...) et jusqu'à la mise en page et l'illustration, voire la correction des épreuves. ROS94J, par exemple, insiste - après un sous-titre éloquent: «Vive l'étapisme !» - sur le fait que "La production d'une nouvelle comporte plusieurs étapes. Il n'en faut négliger aucune, sous peine d'obtenir un texte de qualité douteuse. » (p. 7)

Une troisième structure reconnaissable est d'ordre essentiellement linguistique. Cette approche, elle aussi assez naturelle, reproduit dans un sens ou dans l'autre (de la partie au tout ou du tout à la partie) l'emboîtement des échelles de construction et d'analyse du discours : mot+phrase+paragraphe+texte. Cette structure est rarement dominante - au sens où elle fournirait à elle seule la trame principale de l'ouvrage mais elle est très présente en tant que sous-dominante, la question du choix des mots fournissant la matière d'un chapitre, celle des phrases un autre...

Une quatrième structuration ("anatomique") se fonde sur l'architecture interne d'un document type, propre au champ d'application considéré, en particulier :

- Pour un article de presse : Titre et sous-titre + Chapô + Attaque + Corps du texte + Chute (+ encadrés, légendes photos...).

- Pour un article scientifique : Titre et mots-clefs + Abstract + Etat de la question (previous findings) + Matériel et méthodes + Résultats + Discussion + Conclusion ( + références, tables et figures).

Naturellement, d'autres structurations, plus anecdotiques, peuvent être rencontrées dans de rares cas : GER95J, dans un ordonnancement plus proche d'un traité de bon usage que d'un manuel professionnel, égrène longuement les impropriétés et solécismes à proscrire, GIR97G adopte simplement un classement numérique, fondé sur l'ordre alphabétique des verbes qu'il utilise, quitte à s'éloigner sensiblement du cours usuel des choses (2.8: Mettre des titres; 2.9 : Mettre en page ; 2.10 : Prendre des notes ; 2.11 Rédiger...), etc. Plus fréquents sont, en revanche, les ouvrages qui paraissent suivre la pensée de leur auteur, sans que l'on puisse vraiment y découvrir une nomenclature explicitable : c'est ce que revendique par exemple CAP92J en présentant son ouvrage comme « une conversation professionnelle étendue » (p.xi).

Il n'en reste pas moins que les structures que nous avons discernées plus haut rendent compte de la majorité des ouvrages, et de l'essentiel de la part de leur contenu spécifiquement consacré à la rédaction. Étant cependant entendu qu'elles ne sont jamais utilisées sous une forme aussi pure que ces archétypes pourraient le suggérer, et qu'elles se combinent généralement - par inclusion, juxtaposition, ou par un panachage plus confus - pour donner le texte final. 


\section{Les limites pédagogiques et opératoires des structurations}

Si nous nous attardons sur ces structures types, c'est en partie parce qu'elles permettent, au prix d'une certaine simplification, de distinguer des régularités dans la composition d'un ensemble qui, sans cette lecture, pourrait ne sembler qu'hétérogène Mais c'est aussi parce que cette question est didactiquement capitale, que ce soit pour un manuel ou pour un cours présentiel. Elle conditionne en effet, non seulement la possibilité de retenir la substance des notions ou préceptes rencontrés, mais aussi la possibilité de combiner et d'instancier ultérieurement ces notions ou préceptes pour les adapter aux situations rencontrées dans la vie active.

La chose la plus fondamentale que l'on puisse sans doute dire de la mémoire humaine, au terme d'un siècle de recherches intensives, est qu'à moins qu'un détail ne soit placé dans un schéma structuré, il est rapidement oublié [...] Ce qu'apporte l'apprentissage de principes généraux ou fondamentaux, c'est d'assurer qu'une perte de mémoire n'impliquera pas une perte totale, que ce qui restera permettra de reconstruire les détails lorsque nécessaire. Une bonne théorie n'est pas seulement le moyen de comprendre un phénomène sur le moment, mais aussi celui de s'en souvenir plus tard. (Bruner, 1960, pp. 24-25, notre trad.)

La «méthodologie» de la communication écrite, telle que nous pouvons la percevoir dans le cadre de ce corpus, propose-t-elle un «schéma structuré »? La question est évidemment double, selon que l'on se place du point de vue de la discipline (s'agit-il d'un ensemble de connaissances "structuré »?) ou du point de vue du bénéficiaire (lui permet-on d'acquérir un savoir "structuré »?). Toutefois, l'une et l'autre achoppent sur les mêmes points. Dès lors que l'on ne peut tout retenir, il convient de retenir l'essentiel, ce qui doit être mobilisé au moment d'écrire et qui donne du sens au reste, mais l'idée même d'un essentiel et donc d'un accessoire suppose une hiérarchisation, donc une structuration globale des connaissances. Or, on peut aisément constater que les structures-types que nous avons tenté de distinguer sont difficilement assemblables au sein d'un modèle unique, pour la simple raison qu'elles ne sont pas de même nature. En effet, la structuration "par genres" (type 1) part des normes admises (formes canoniques), la structuration "par étapes" (type 2) part du rédacteur lui-même (processus), et la structuration "linguistique" (type 3) part de la langue. De surcroît, la structuration "anatomique" (type 4) est à la fois profondément semblable à celle des genres (dans la mesure où elle part du produit) et radicalement opposée dans son principe (dans la mesure où elle se fonde sur une forme générique, quand les genres multiplient au contraire les spécificités). 
De ce fait, toute tentative de structuration est confrontée - généralement sans que les auteurs semblent s'en être avisés - au défi qui consiste à guider de leur mieux leurs lecteurs-élèves dans un dédale de notions incommensurables, qui évoquent souvent la taxonomie de Borges (cit. in Foucault, 1966, p. 7) :

Les animaux se divisent en a) appartenant à l'empereur, b) embaumés, c) apprivoisés, d) cochons de lait, e) sirènes, f) fabuleux, g) chiens en liberté, h) inclus dans la présente classification, i) qui s'agitent comme des fous, j) innombrables, $\mathrm{k}$ ) dessinés avec un pinceau très fin en poil de chameau, l) et cetera, m) qui viennent de casser la cruche, n) qui de loin semblent des mouches.

Le cas des genres - donc des préceptes fondés sur les usages et normes socioprofessionnels - mérite que l'on s'y arrête comme particulièrement révélateur. C'est en effet là que se retrouvent les plus fortes contradictions internes, mais aussi les plus grandes différences entre les divers auteurs. Près de la moitié de ceux-ci détaille, souvent très longuement, les différentes formes qu'il convient de respecter, quand l'autre moitié se dispense purement et simplement de les aborder. Cette séparation transcende à nouveau la question des domaines d'application : dans le cas du journalisme, la description des genres constitue la partie la plus importante de plusieurs manuels (ANT95J, par exemple, lui consacre 71 pages, soit 15 chapitres sur 24) tandis que plusieurs autres, comme BLU88J ou HER79J ne les évoquent pas du tout. De même, un ouvrage généraliste comme COL92G est presque intégralement constitué d'une liste de formes canoniques (du faire-part, p. 74, au communiqué de presse, p. 276) tandis que RIC92G, non moins généraliste, ne les évoque jamais. Comment une telle question peut-elle être aussi importante pour certains, et l'être aussi peu pour d'autres ?

Le recours à des genres naturalisés est, certes, séduisant à plus d'un titre. Il l'est pour les auteurs/enseignants, qui peuvent, par induction, produire à bon compte de la connaissance enseignable (voire publiable), au risque de se voir opposer la tirade de Dorante :

«Vous êtes de plaisantes gens avec vos règles dont vous embarrassez les ignorants et nous étourdissez tous les jours. Il semble, à vous ouïr parler, que ces règles de l'art soient les plus grands mystères du monde; et cependant ce ne sont que quelques observations aisées que le bon sens a faites (...)» (Molière, 1835, p. 497).

Il est également séduisant pour les lecteurs/étudiants, qui se trouvent ainsi dotés de modèles clairs et, en apparence, aisés à reproduire.

Toutefois, l'érection du produit final en norme de production, en tout cas quand elle est dominante s'avère difficilement tenable. Outre le fait qu'elle est généralement assez rigide, voire péremptoire et, par nature, extrêmement conservatrice (puisque 
« ce qu'il faut faire » découle de «ce que l'on croit qu'il est d'usage de faire » plus que de la compréhension profonde de ce que l'on cherche à faire), il est évident qu'elle ne permet pas en soi de structurer de façon cohérente les fondements de la rédaction appliquée. Elle ne le permet pas pour le rédacteur :

Le rédacteur ou la rédactrice professionnelle ne peut faire l'économie de cette question fondamentale et ce n'est pas en limitant son savoir à des connaissances de type procédural sur les genres d'écrits ou sur la lisibilité des textes que ce questionnement prendra forme. Au contraire, cette limitation de son champ de compétences à la dimension procédurale de l'écriture encourage une conception normativiste et rassurante de l'acte d'écrire, qui apparaît ainsi réductible à un ensemble de règles prédéfinies qui se substitueraient à la nécessité de réfléchir et de faire des choix. Cette vision est contraire à la réalité de la rédaction professionnelle: le choix des bonnes stratégies textuelles, discursives et stylistiques et leur amalgame original résulte d'une compréhension de fond des liens entre les mécanismes de production de sens dans le langage et le sens que prennent les discours organisés dans leurs formes différentes. (Beaudet, 1999)

Elle le permet encore moins pour les ouvrages eux-mêmes, puisque les mêmes indications doivent alors être répétées dans des parties concernant des genres différents. Ainsi, dans COL92G, la question de la longueur des phrases est-elle évoquée à deux reprises à 15 pages d'intervalle, toutes deux assez loin du début de l'ouvrage: "Écrire des phrases très courtes. Il n'est pas forcément nécessaire d'ajouter des qualificatifs. » (p. 148) "Il existe des critères de lisibilité : faire des phrases courtes; employer des termes simples; rechercher l'expression exacte. » (p. 163). On la trouve également p. 241 ( Des phrases courtes [...] plus courte est la phrase, meilleure est la compréhension»), et ailleurs, encore. Le premier extrait se situe dans une partie consacrée à la rédaction des affichettes, le second est relatif à la rédaction des rapports, le troisième porte sur les notes de service. On ne trouve en revanche aucun conseil de ce type dans la partie consacrée aux circulaires, qui détaille en revanche le «tract», la «circulaire privée» et la «circulaire administrative». Ainsi le traitement de la question de la longueur des phrases se trouve-t-il à la fois redondant (globalement) et lacunaire (localement). Plus généralement, la séparation des différents genres, quand elle est structurellement dominante supposerait qu'il y ait une façon spécifique d'écrire des notes de service, différente de celle des circulaires ou des rapports, ce qui reste à démontrer.

Un autre effet curieux de l'approche par les genres est qu'elle conduit tout naturellement, par une tendance naturelle à l'exhaustivité, à présenter aux apprentis rédacteurs des formes auxquelles la plupart d'entre eux ont bien peu de chances d'être confrontés à bref délai, comme l'éditorial, le brevet, ou encore le rapport annuel destiné aux actionnaires (avec un exemple tiré du rapport d'Usinor). Naturellement, ces développements ne sont pas gênants par eux-mêmes, mais ils 
contribuent à diluer les conseils dispensés, et à noyer le lecteur sous une profusion de détails et de définitions.

Enfin, et surtout, la référence à des genres ou des documents-types, par nature normative (voire conservatrice), prend nécessairement modèle sur des produits archétypiques, qui ne correspondent donc pas nécessairement aux réalités professionnelles. Ainsi tous les articles de recherche, même en sciences expérimentales et même (surtout?) écrits par des chercheurs réputés ne se coulent pas dans le moule épuré, si ce n'est un peu idéalisé, présenté dans les cours de communication scientifique. De même, comme s'en inquiète un enseignant en journalisme de l'Université de New-York, "les étudiants en journalisme travaillent essentiellement avec les formes d'écriture les plus simples, et les plus figées ${ }^{51}$, des formes que la plupart de leurs professeurs n'ont pas utilisées depuis longtemps, et, pour dire la vérité, n'ont aucun désir de réutiliser. 》 (Stephens, 2010).

De façon générale, le rapport entre genres enseignés et genres pratiqués peut être assez marqué dans certains cas (par exemple celui des communiqués de presse ou, à la rigueur, celui des lettres de publipostage) mais bien d'autres familles de textes, et non des moindres (les annonces publicitaires, les écrits de vulgarisation, les blogues, les reportages, etc.) sont si éloignés de toute norme que l'idée même de recourir à des moules canoniques semble tenue en échec.

Il n'entre évidemment pas dans notre propos de contester la nécessité d'apprendre aux futurs professionnels certaines spécificités - réelles ou imaginaires - des différents documents qu'ils pourront être amenés à produire, mais on doit insister sur le fait que cette particularisation, somme toute assez triviale et essentiellement descriptive, s'inscrit nécessairement dans une alternative entre deux modèles implicites d'apprentissage (Figure 5).

\footnotetext{
${ }^{51} \mathrm{~L}$ 'auteur vise ici l'attaque récapitulative (ou $5 \mathrm{~W}+\mathrm{H}$ ) et la construction en pyramide inversée, qui sont encore présentées comme la forme parfaite de l'écriture d'information, alors que de nombreuses études ont montré qu'il n'en était rien.
} 


\section{a) Conception " binaire "}

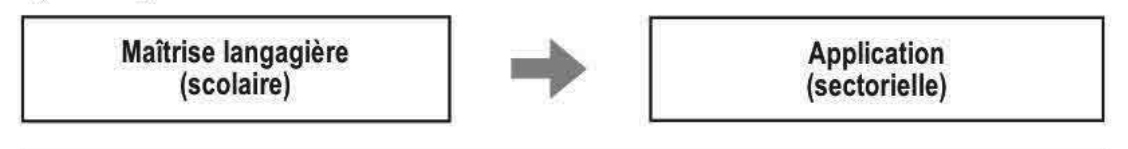

\section{b) Conception " ternaire »}

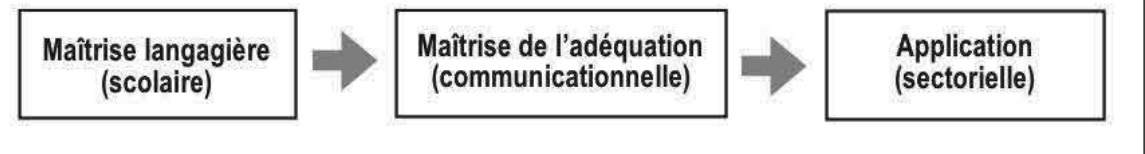

\section{FIGURE 5}

\section{Représentation heuristique des modèles d'apprentissage pratique de la communication écrite}

S'il ne s'agit que de couler son expression dans des moules professionnels éprouvés, il est légitime de passer directement du scolaire au spécifique, mais du même coup, la référence au destinataire devient inutile (la transition se fait d'une norme langagière à une norme documentaire), alors même que, comme on l'a vu, cette référence - « écrire pour son lecteur 》 - était le principal point de convergence des discours des divers auteurs. D'autre part, la transition directe entre ce qui relève de l'apprentissage scolaire et ce qui relève de l'entraînement aux usages sectoriels met en question la pertinence même d'un enseignement «supérieur» en communication, ou, du moins sa valeur ajoutée (par rapport à l'école, d'un côté, et aux praticiens, de l'autre) et sa capacité à conférer une réelle expertise.

On peut toutefois émettre l'hypothèse que la prépondérance du modèle binaire (langue + particularisation) relève moins d'une stratégie didactique assumée que d'un pis-aller : en l'absence de solides connaissances appliquées sur les logiques et les facteurs de l'adéquation communicationnelle - donc en l'absence de la pièce centrale du dispositif - il semble difficile d'éviter une telle juxtaposition, au prix des redondances ou incohérences observées plus haut.

Cette hypothèse peut être testée en analysant les inter-influences des livres du corpus à partir de leurs citations. Globalement, ces ouvrages se citent assez peu entre eux et, lorsqu'ils le font, tendent plutôt à demeurer dans leur spécialité sectorielle. On peut toutefois distinguer quelques auteurs ou groupes d'auteurs «influents», 
voire «centraux »: alors que beaucoup d'autres ne sont jamais mentionnés, sept d'entre eux (soit $14 \%$ de l'ensemble des membres du corpus) totalisent 70 mentions sur 113 (soit $62 \%$ ). Or, à l'exception d'un titre assez spécifique ${ }^{52}$, tous les ouvrages « influents » présentent, indépendamment de leurs différences de date, de continent, de langue ou de spécialité, des caractères très similaires que les autres ne comportent pas, ou moins: tous s'avèrent en effet centrés sur l'adéquation au destinataire (notamment les questions de «lisibilité ", d'« intérêt humain », etc.) s'efforçant donc de réaliser le projet fondateur du domaine. De ce fait, aucun n'est structuré en genres, ce qui ne signifie naturellement pas qu'une telle question est pour eux sans utilité dans l'absolu, mais simplement que ces auteurs ont choisi de concentrer leur approche sur les fondements et les logiques de la communication, plutôt que sur le détail formel de ses concrétisations particulières.

Cependant, le petit groupe des ouvrages «influents» ne résout pas, en l'état, le problème du «tronc commun» communicationnel qui relierait les aspects langagiers et les particularisations sectorielles. En effet, chacun d'entre eux se donne à lire comme une position particulière - assez idiosyncrasique, bien que se référant volontiers à des travaux empiriques - et se présente soit comme un exposé « au fil de la pensée », soit comme une combinaisons ad hoc de structuration linguistique (mot, phrase...), organique (titre, introduction...) et de considérations sur l'attitude du rédacteur, les attentes du lecteur, etc. Ainsi, ces ouvrages soulignent-ils, plus encore que les autres, le manque de paradigmes unificateurs susceptibles de donner pleinement à ce domaine sa cohérence académique, sa pertinence pédagogique et son efficacité opératoire.

\section{De la microstructuration à la macrostructuration}

Il parait, a priori, audacieux d'esquisser un rapprochement entre des manuels de communication écrite - dont l'appellation même de "manuels » appellerait des développements trop longs ici $^{53}$ - et l'enseignement de la communication en général, tant les différences de toute nature sont évidentes. Pour n'en mentionner qu'une, l'enseignement universitaire vise à conférer, au delà des aptitudes techniques, un

\footnotetext{
${ }^{52}$ Il s'agit du guide de rédaction d'une célèbre école de journalisme française, qui a bénéficié à ce titre d'une visibilité particulière, et, au fil de ses nombreuses rééditions, d'une diffusion particulièrement large.

${ }^{53}$ Le terme, utilisé ici dans un sens générique, recouvre comme on l'a dit des ouvrages associés à des cours universitaires et des guides pratiques résolument athéoriques, mais ni les uns ni les autres ne peuvent réellement s'assimiler à des manuels scolaires ou des médias pédagogiques, dont la classification et l'analyse sont elles-mêmes problématiques (Choppin, 2008 ; Bruillard 2005 ; Moeglin, 2005) mais qui, du moins, ont une vocation, un public et un rapport aux connaissances disciplinaires nettement plus homogènes en comparaison.
} 
recul critique et analytique dont les ouvrages analysés sont, pour la plupart, dépourvus (ce en quoi on notera qu'ils convergent avec la plupart des modules de formation continue privés ou même publics).

Pour autant, tout cursus en communication peut s'approcher, comme un discours ${ }^{54}$ global résultant de l'assemblage de différents contenus dont l'échelle et la nature sont hétérogènes. Ce caractère composite, largement accentué par le système des crédits, lui confère à la fois un abord électif (les étudiants peuvent, dans une certaine mesure, choisir les «parties» auxquelles ils accèdent ou l'ordre dans lequel ils les parcourent) et une expérience linéaire (du premier jour de cours de première année jusqu'à la fin du dernier cours) au terme de laquelle ils seront censés avoir acquis les aptitudes recherchées.

Dans cette perspective, plusieurs questionnements relevés en observant les manuels pourraient contribuer à la réflexion pédagogique sur les parcours en communication.

Le premier est celui de la spécificité des champs d'application spécialisés, en particulier la rédaction scientifique et technique, dont on a vu que les distinctions revendiquées, si elles présentaient a priori des justifications pratiques évidentes, n'étaient, pour l'essentiel, soutenues - au-delà de la posture des auteurs - que par la différence d'une partie des textes étudiés, tout en demeurant en termes de contenu et de structure, très proches des ouvrages visant d'autres spécialités ${ }^{55}$. Or, les revendications d'autonomie que l'on peut rencontrer dans les manuels examinés par exemple : «la prise en charge de l'information scientifique par les scientifiques eux-mêmes est un gage du succès de la vulgarisation » (LAZ93S, p. 118) - ne se retrouvent pas seulement dans des livres portant sur d'autres domaines, non représentés dans le corpus, tels que la communication administrative: "l'Administration a et aura généralement "une manière particulière d'écrire et d'exprimer sa pensée" » (Catherine, 1996, p.10). Elles sont également communes dans les propos ou attitudes concernant l'enseignement universitaire. Ainsi, des chercheurs en sciences expérimentales peuvent-ils affirmer à propos de la formation des étudiants que "les savoir-faire en communication qu'il faut développer devraient venir de - et être intégrés à - la discipline, plutôt qu'être présentés séparément. » (Radloff, de la Harpe \& Zadnik, 1996, notre trad.), au risque de

\footnotetext{
54 On considère ainsi qu'une somme de discours - les cours - dont chacun peut être porteur de points de vue spécifiques (implicites ou explicites), n'en constitue pas moins un discours global, indépendamment des contradictions internes que la multiplicité des locuteurs et des matières ne peut manquer d'engendrer.

5 Même dans le cas du journalisme, dont la spécificité axiologique et procédurale suffisamment indéniable pour justifier sa distinction, une grande partie des préceptes opératoires rencontrés s'avéraient communs à l'ensemble de la rédaction professionnelle, tandis que bien des ouvrages de communication d'entreprise s'inspiraient explicitement de procédés journalistiques (comme la pyramide inversée, évoquée plus haut).
} 
méconnaître non seulement les dimensions opératoires dépassant les préceptes superficiels, mais aussi quatre décennies de recherches éclairantes sur les logiques, contraintes, problèmes épistémologiques, sociaux, etc., de la communication des sciences (menées pour l'essentiel par des chercheurs en communication). Notons que la question de la spécificité s'est, par ailleurs, intensifiée avec l'essor des nouvelles technologies de communication, qui s'est accompagné de fréquentes affirmations sur le caractère radicalement nouveau et différent des aptitudes nécessaires, y compris dans le cas de la communication écrite (la lecture d'une page web étant réputée - contre toute plausibilité psycholinguistique - foncièrement dissemblable de celle d'une page imprimée).

Pour autant, les sciences de la communication sont-elles réellement en mesure de proposer, face aux besoins issus de différents types ou secteurs d'application, un contenu pédagogique prévalant non seulement par son raffinement théorique mais aussi, voire surtout, par son efficacité concrète ? C'est sans doute ici que se pose le principal problème structurel analysé plus haut, celui consistant à ordonnancer mais cette fois-ci à l'échelle d'un cursus - les différents constituants afin d'offrir un «schéma structuré» (Bruner, 1960) qui permette non seulement l'assimilation, la mise en relation et la mémorisation durable des notions abordées, mais aussi leur adaptation ultérieure à la résolution stratégique des problèmes rencontrés dans la vie professionnelle. Or, on l'a dit, les manuels procèdent essentiellement par inclusion et juxtaposition, évoquant la célèbre charge de Sarcey contre cet "amas informe, indigeste de petits faits, qui tombent les uns par-dessus les autres, sans qu'aucun ferment d'idées mette en jeu et fasse lever cette pâte coupée en lourdes tranches. ${ }^{56}$ (cit. in Brisson, 1904, p. 63).

Que la formation supérieure type soit, elle aussi, coupée en tranches - certaines assez lourdes, à en croire les étudiants, d'autres forts légères quand elles ne reposent que sur des préceptes et exercices pratiques - est, comme on l'a rappelé, une nécessité organisationnelle. Mais celle-ci n'en tend pas moins à faire, dans une certaine mesure, reposer sur les étudiants la charge de construire eux-mêmes l'expertise qui leur sera nécessaire, en retenant ici et là des éléments de savoirs savants et de techniques opératoires, et en reliant comme ils le peuvent ces fragments pour qu'ils se donnent mutuellement du sens. L'expertise, en effet, ne découle ni du savoir scientifique ni de l'habileté pratique : à la confluence de ces deux pôles, elle en constitue un troisième, celui de la compétence approfondie, orientée vers la résolution de problèmes concrets mais enrichie et éclairée par des connaissances théoriques solides.

\footnotetext{
${ }^{56}$ On se souvient que Sarcey s'en prenait ici au journalisme de nouvelles de Girardin, dont, selon lui, ne se dégageait «pas une pensée juste, pas une vue d'ensemble, pas même une observation de détail » (ibid., p. 63)
} 
Une chose qui distingue un professionnel d'un artisan est la connaissance de la théorie, des principes sous-jacents qui font que les techniques fonctionnent. Un savoir-faire s'acquiert par mimétisme, en regardant un maître agir et en imitant cette personne. Une profession s'acquiert à partir des principes fondamentaux, de façon à ce que, lorsque les choses changent, le professionnel comprenne ces changements et ajuste ses techniques en conséquence. (Meyer, 1996)

Ce qui vaut pour le champ des médias, objet du propos précédent, est tout aussi vrai pour l'ensemble du spectre de la communication, comme l'illustre éloquemment l'exemple rapporté par un ancien président de la Society for Technical Communication :

Un chef de projet confiait " j'ai fait une recommandation sur celles des informations qu'il fallait mettre en ligne et celles qu'il convenait d'imprimer, et je sais dans mes tripes que c'est une bonne recommandation." Cette recommandation fondée sur l'instinct ou les préférences est l'art de la communication technique. Elle continuait « je n'ai pas la moindre chose pour appuyer mes recommandations ». Elle faisait allusion à des résultats d'études et recherches indiquant quel média utiliser dans certaines situations. Des décisions fondées par la recherche forment la science de la communication technique. Les professionnels en exercice veulent se sentir à l'aise avec ce qu'ils recommandent, et la recherche peut contribuer à cette confiance. (Carliner, 1995, souligné par nous)

Il n'est pas indispensable d'adopter toutes les convictions épistémologiques de cet auteur pour constater que la question de l'intégration des connaissances de référence aux apprentissage opératoires, intégration dont on a constaté l'échec dans le cas des manuels, n'est pas forcément mieux résolue dans l'organisation des programmes universitaires : où sont donc - pour reprendre les termes de Meyer - ces «principes fondamentaux » et comment irriguent-ils l'«acquisition par mimétisme » assurée par les cours pratiques?

Juxtaposer des cours théoriques et des enseignements pratiques, de même que multiplier les cours spécialisés - réseaux sociaux, traitement de l'image ou du son, communication avec les personnes âgées, les jeunes, les investisseurs, les minorités ethniques... - au fur et à mesure que de nouveaux besoins se font jour, ou que les professeurs s'intéressent à de nouvelles problématiques - peut repousser le problème, mais plus difficilement le résoudre (quelles que soient la pertinence et la qualité de chacun des cours anciens ou nouveaux ainsi agrégés). En effet, l'enseignement universitaire n'en demeure pas moins confronté à une alternative structurale qui, mutatis mutandis, se trouve être la même que celle relevée plus haut dans les ouvrages. De même que ceux-ci, face à la difficulté de rapprocher des aspects de portée générale (notamment linguistiques) et des genres textuels sectoriels, recouraient, en l'absence d'un «tronc commun communicationnel» 
central, à un assemblage binaire, de même, l'assemblage de cours (historiques, sociologiques...) de portée générale et de cours pratiques fondés sur des usages sectoriels ne permet guère de distinguer les articulations et même le centre de gravité, de l'ensemble ainsi construit: le modèle implicite d'apprentissage binaire est sans doute déplacé par rapport à celui des manuels, mais il n'est pas foncièrement différent (Figure 6).

a) Conception " binaire "

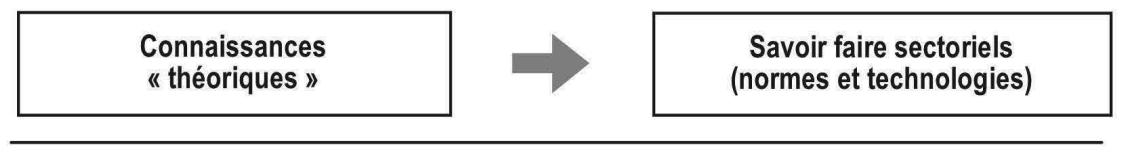

b) Conception " ternaire "

\begin{tabular}{|c|c|c|c|}
\hline $\begin{array}{c}\text { Connaissances } \\
\text { «théoriques » }\end{array}$ & $\begin{array}{c}\text { Intelligence de l'adéquation } \\
\text { (communicationnelle) }\end{array}$ & $\begin{array}{c}\text { Savoir-faire } \\
\text { (sectoriels) }\end{array}$ \\
\hline Savoir & Expertise & Pratique \\
\hline
\end{tabular}

\section{FIGURE 6}

Représentation heuristique des approches pédagogiques de la formation universitaire professionnalisante en communication

\section{Les problèmes pédagogiques sont des problèmes épistémologiques}

Si l'étude des caractères communs des manuels de rédaction tend, par essence, à niveler leurs spécificités (et, en particulier, à amalgamer des auteurs - souvent les plus péremptoires - dont les compétences s'avèrent pour le moins discutables et d'autres témoignant d'une réflexion beaucoup moins superficielle), il va sans dire que prétendre évoquer globalement les formations post-scolaires à la communication est encore plus problématique. Même en laissant de côté les pourvoyeurs privés de formations courtes, et les programmes universitaires d'autres disciplines incorporant des cours de "communication », de façon à restreindre le propos aux seuls cursus relevant des sciences de la communication, il demeure difficile d'aborder ces derniers comme un ensemble homogène. La réflexion didactique et pédagogique, par exemple, est beaucoup plus avancée dans certains cours et certains départements que dans d'autres. Mais quel que soit le soin avec lequel un «bouquet » de cours est rassemblé et érigé en programme, cette construction n’en demeure pas moins limitée 
dans sa cohérence et son efficience par des fragmentations ou des oppositions fondamentales.

La première est celle du rapport aux professionnels. Les sciences de la communications sont, plus que la plupart des autres domaines académiques (droit, éducation, sciences politiques, etc.) confrontées au problème de posture résultant, d'un côté, d'une distanciation critique bien établie, voire d'une répulsion instinctive face à toute instrumentalisation, et, de l'autre, du désir ou de la nécessité de former à des emplois, notamment dans les entreprises commerciales du secteur de la communication et des médias. Mais, paradoxalement, cette distanciation peut conduire jusqu'à l'abdication, les chercheurs supposés - comme dans d'autres disciplines - éclairer les professionnels se retrouvant au contraire en position de demander à ces derniers ce qu'ils doivent enseigner (à l'échelle du programme) ou leur déléguant cette tâche (à l'échelle du cours). Le trait est évidemment un peu forcé, dans la mesure où le dialogue avec les professionnels et leur contribution à l'enseignement, voire à la recherche, constitue une source d'enrichissement indiscutable, voire indispensable. De surcroît, les stages au sein des organisations constituent un complément pédagogique particulièrement précieux. Mais il ne s'ensuit pas que les praticiens, et notamment les associations d'employeurs, soient forcément les plus clairvoyants (et les plus impartiaux) en ce qui concerne les évolutions de leur domaine, les attentes de leurs publics, et jusqu'à leurs propres besoins réels en termes de compétences ${ }^{57}$. Ceci ne concerne pas seulement l'échelle de la prospective économique et sociale ou celle des ressources humaines : sur le plan strictement technique, l'étude des manuels a montré que ceux qui étaient l'œuvre de «purs » praticiens comptaient souvent parmi les plus discutables sur le fond mais aussi en pratique.

La seconde dichotomie, sœur de la précédente, est celle qui oppose constamment la « théorie » et la pratique. On sait combien les avis des professeurs et des étudiants peuvent différer sur ce point, ainsi que l'illustre Carliner, qui, comme praticien et enseignant, connaît les deux côtés de la barrière :

Les professeurs veulent fournir à leurs étudiants un substrat théorique. Toutefois, la théorie que la sphère académique valorise est souvent de la théorie pour ellemême. [...] Beaucoup d'étudiants sont plus pragmatiques ; ils veulent seulement apprendre comment accomplir les tâches auxquelles ils aspirent. Ils comptent apprendre des habiletés, essentiellement techniques comme la P.A.O., le développement de contenus en ligne ou la programmation en $\mathrm{C}$ - et utiliser

\footnotetext{
${ }^{57}$ Il suffit, comme l'auteur de ces lignes, d'avoir siégé dans des comités mixtes ou des organismes d'études sectoriels, pour constater à quel points les souhaits exprimés peuvent être déterminés par des considérations à courte vue ou orientés par des convictions peu fondées.
} 
diverses technologies de communication telles qu'Internet. Ce sont les aptitudes que les étudiants voient détailler sur les annonces d'emploi, et, de ce fait, ce sont celles dont ils croient avoir besoin pour réussir dans le monde du travail. (Carliner, 1995, p. 548)

Cependant, comme le note cet auteur, les compétences de ce type ne sont mentionnées dans les annonces qu'en raison de leurs spécificités : ce ne sont pas les seules nécessaires pour réussir dans un emploi communicationnel. Les capacités générales à comprendre, à analyser, à apprendre, à exprimer, etc., s'avèrent au moins aussi importantes. En somme, le risque que peut engendrer une focalisation trop exclusive sur les secteurs et les techniques, émergentes ou non, est celui qu'il y aurait à vouloir former des spécialistes qui ne seraient pas, auparavant, des généralistes. Mais qu'y a-t-il de suffisamment "général » pour être commun à tous les avatars de la communication, sans être pour autant «général» au point de se retrouver dans n'importe quel enseignement universitaire (de la prise de note à la pensée critique) ?

En l'absence de paradigmes unificateurs, les savoirs en communication forment avant tout une culture interprétative - ce que Miège (1995) appelle la «pensée communicationnelle» - riche de plus d'un demi-siècle d'approches multiréférentielles, dont les dissensions théoriques mêmes apportent une grande acuité analytique des situations de communication. Et cette culture, sous une forme plus ou moins condensée, peut tant bien que mal se transmettre : la plupart des programmes n'y manquent pas et les ouvrages destinés aux étudiants de premier cycle abondent. En revanche - et c'est bien là que le bât blesse - cette culture est extraordinairement difficile à mettre au travail. Comme bagage professionnel, elle excelle à dissiper les illusions (ce qui n'est déjà pas si mal), mais se refuse absolument à conseiller ou à prescrire, et plus encore à promettre quelque efficacité que ce soit. D'où, sans doute, son absence frappante des manuels de communication écrite: comme on l'a remarqué supra, le seul élément qui puisse en relever (à moins d'inscrire la PNL dans la descendance légitime de l'école de Palo Alto) est le modèle télégraphique de Shannon et Weaver, c'est-à-dire celui-là même contre lequel se sont construits la plupart des discours savants dans le champ de la communication.

Les sciences de la communication, à l'inverse de beaucoup d'autres disciplines, sont avares de connaissances déclaratives et de modèles de moyenne portée intégrables à une réflexion praticienne orientée vers l'action, notamment parce qu'elles n'ont pas ou peu de tradition de recherche appliquée (en tout cas depuis les fonctionnalistes américains ou, en France, l'épisode d'Abraham Moles) et que la légitimité même de telles recherches y demeure plus que problématique. D'où les bricolages conceptuels hasardeux que l'on peut rencontrer dans des domaines comme la publicité (Bouzon, 2006), le journalisme (Winkin, 1996) et la communication en général, qu'elle soit écrite (Labasse, 2009) ou «non verbale» (Lardelier, 2008). 
On ne s'attardera pas sur les causes et effets de cette coupure entre le champ des recherches et celui des pratiques, qui engage d'assez longues considérations (pour une exploration de ses déterminants historiques, manifestations sectorielles, enjeux épistémologiques et idéologiques, particularismes continentaux, etc., voir Labasse 2012). L'essentiel ici est de relever que, si une telle séparation présente des inconvénients scientifiques et praxéologiques (Loneux, 2004 ; Mercier 2007), c'est sur le plan pédagogique que ses conséquences sont les plus directes et les plus sévères. Si l'on peut relever ça et là des tentatives pour réduire la distance entre théories et pratiques (par exemple Dainton et Zelley, 2005; Dagenais, 2006 ; Boistel, 2007...) s'exposant parfois à des critiques vigilantes il n'en reste pas moins que l'espace entre les recherches et les méthodes opératoires demeure pour l'essentiel en friche, et que cette friche, se situant entre théories et pratiques, se trouve au centre de l'espace pédagogique que dessinent les cursus en communication.

C'est précisément en ceci que le rapprochement entre les manuels de rédaction et les formations en communication ne relève pas d'un simple rapport d'analogie mais bien d'un rapport de concentricité. Archétypes de l'aspiration à l'«ars bene dicendi » au cœur de toute pratique communicationnelle, ces ouvrages pratiques sont les plus frontalement exposés aux lacunes de ce dernier. Non pas aux lacunes des connaissances disponibles (le problème d' "écrire pour son lecteur» aurait par exemple beaucoup à retirer des théories de la réception et de la psycholinguistique pragmatique, pour ne citer qu'elles), mais à la défaillance des connexions entres celles-ci et les problématiques opératoires. D'où le même inconfort pour structurer un livre ou un programme, dès lors que le tronc qui pourrait en unir les branches (et même les racines théoriques) n'est pas en mesure d'assurer la solidité de l'ensemble.

Pour terminer, il paraît tentant de se risquer, à partir du même centrage, à élargir la perspective en adoptant non plus l'échelle d'un savoir-faire (celle d'un manuel ou d'un cours), ni celle d'un apprentissage complet (celle d'un programme), mais celle, globale, de l'apprentissage post-scolaire de la communication en général et de ses rapports avec la discipline - ou l'interdiscipline - du même nom.

En effet, comme on l'avait rappelé plus haut, le développement et la diversification de ce secteur économique tend à multiplier les domaines d'application, les demandes de formation, mais aussi les pourvoyeurs de formation de tous niveaux et de tous horizons. D'où le risque ressenti d'une dynamique centrifuge de cet ensemble, dont la croissance et les particularisations ne cesseraient de diluer la substance et la cohérence. Toutefois, une telle tendance ne pourrait qu'être sérieusement accentuée par le manque, sinon d'attraction, du moins d'attractivité, du centre de cet agglomérat pour sa périphérie. La nécessité de références communes ne se décrète pas ex cathedra; elle ne peut se fonder que sur le sentiment que ces références sont « utiles », non seulement à l'analyse, mais aussi à la maîtrise réfléchie des stratégies discursives, si spécifiques que puissent être 
leurs buts, leurs domaines ou les médias et technologies auxquels elles peuvent avoir recours.

Ainsi semble-t-il difficile d'attribuer avec fatalisme la dispersion des domaines et des formations aux seuls vents des changements techniques et des appétits économiques: elle est aussi le fruit de postures épistémologiques, voire axiologiques, qui, pour légitimes qu'elles soient, laissent aux sciences de la communication une part notable de la responsabilité de cet éloignement, que l'on juge celui-ci souhaitable ou non (il existe de bons arguments dans les deux sens).

\section{Conclusion}

Depuis deux millénaires et demi, les communicateurs et, surtout, ceux qui les forment, sont confrontés au défi de justifier les savoirs sur lesquels se fondent leur tecknè, et même de structurer leur discours à partir de ces savoirs :

...il est au contraire évident que pour enseigner à discourir avec art, il faut savoir exactement indiquer l'essence de la chose à laquelle se rapporte l'art de la parole ; cette essence, c'est l'âme. [...] Il est donc évident que Thrasymaque, ou tout autre qui voudrait enseigner avec soin l'art de la rhétorique devra d'abord, avec toute l'exactitude possible, décrire l'âme [...] Il décrira en second lieu, comment et sur quoi elle agit, comment et par quoi elle peut être affectée [...] (Platon, 1922, p. 270-271)

Le bilan contemporain que, dans la même tradition, l'on peut esquisser à partir de "ce qui est écrit dans les livres sur l'art de discourir» (Platon, 1922, p. 266) ne permet pas d'affirmer que cette double question ait sensiblement progressé au cours de tant de siècles.

Il est vrai que le développement de l'expertise instrumentale n'a guère été au centre des préoccupations des sciences de la communication modernes. Mais en matière de communication, les problématiques scientifiques et les problématiques pédagogiques coïncident souvent mal, et ce qui peut être perçu comme insignifiant dans le premier cas ne l'est pas forcément dans le second.

C'est ce que la présente étude, bien qu'envisagée moins comme une démonstration formelle que comme une proposition (ou une invitation au débat), visait à examiner.

La difficulté éprouvée par les manuels - par ailleurs bien pourvus en préceptes, avis et normes génériques ${ }^{58}$ de toute sorte - à fonder et structurer un discours répondant au projet même par lequel ils se définissent (" écrire pour son lecteur »), a pu être interprétée comme le signe des carences touchant précisément les 
connaissances nécessaires à ce projet central, ce que l'analyse de leurs citations mutuelles tendrait à confirmer.

Le problème consistant à construire un programme d'enseignement à partir de cours de nature et de finalité très variées de façon à former un tout cohérent et orienté vers la maîtrise réfléchie des stratégies discursives - ou, pour emprunter les termes de Socrate, à " diriger toutes ces choses vers un but commun », sauf à laisser aux étudiants "le soin de se tirer eux-mêmes d'affaire sur ce point » (Platon, 1922, p. 107) - nous a paru se heurter à des difficultés d'interconnexions similaires à celles des manuels, quoique différentes dans leurs manifestations.

Dans l'un et l'autre cas, en effet, se manifeste le problème d'une expertise réelle de la communication, au confluent des connaissances savantes et des problématiques praticiennes. Or les insuffisances de celle-ci n'affectent pas que les manuels pratiques, ni même la cohérence pédagogique des programmes universitaires. À une plus large échelle encore, elles interrogent les sciences de la communication sur leur désir de se situer durablement comme le lieu de connaissance et de formation de référence pour les professions dont elles analysent les discours et les pratiques. 


\section{Bibliographie}

Beaudet, Céline (1999). Les compétences linguistiques et discursives du rédacteur professionnel : un ensemble à circonscrire. In C. Guével et I. Clerc. (Eds.), Les professions langagières à l'aube de l'an 2000 (pp. 3-18). Ste Foy: Ciral Université Laval.

Boistel, Philippe (2007). Gestion de la communication d'entreprise, aspects théoriques et pratiques. Paris : Éditions Lavoisier.

Bouzon, Arlette (2006). Modèles et conception publicitaire. Des pratiques incertaines, entre bricolage et braconnage. Communication et organisation, 30, 126-146.

Brisson, Adolphe (1904). Portraits intimes (1894). Paris : Armand Colin.

Bruillard, Éric. (2005). Les manuels scolaires questionnés par la recherche. In Éric Bruillard (dir.), Manuels scolaires, regards croisés. Caen : Scéren/CRDPde Basse-Normandie (pp.13-36).

Bruner, Jerome. (1960). The Process of Education. Cambridge : Harvard University Press.

Catherine, Robert (1996). Le style administratif. Paris : Albin Michel.

Carliner, Saul (1995). Finding a common ground: What STC is, and should be, doing to advance education in information design and development. Technical communication, 42, 546-554.

Chevallard, Yves. (1985). La Transposition didactique ; Du savoir savant au savoir enseigné. Grenoble : La Pensée Sauvage.

Choppin, Alain. (2008). Le manuel scolaire, une fausse évidence historique. Histoire de l'éducation, 117, 7-56.

Dagenais, Bernard (2006). Des modèles utiles mais ignorés : le cas des relations publiques. Communication et organisation, 30, 149-162.

Dainton, Marianne, et Zelley, Elaine D. (2005). Applying Communication Theory for Professional Life: A Practical Introduction. Thousand Oaks: Sage publications.

Craig, Robert T. (1999). Communication Theory as a Field. Communication Theory, 9 (2), 119-161.

Escarpit, Robert (1976). Théorie générale de l'information et de la communication. Paris : Hachette.

Foucault, Michel (1966). Les Mots et les Choses. Paris : Gallimard, 1998. 
Labasse, Bertrand (2006). La communication écrite; Une matière en quête de substance. Lyon : Éditions Colbert.

Labasse, Bertrand (2009). L'écrit professionnel : ambiguïtés et identités d'un objet académique indistinct. Pratiques, 143, 233-248.

Labasse, Bertrand (2012). Un trou noir dans la galaxie : la compétence opératoire dans les recherches en communication. Canadian Journal of Media Studies. 10 (2), 176-214.

Lardellier, Pascal (2008). Arrêtez de décoder ! Une généalogie critique des pseudosciences du "décodage non verbal". Communication \& langages, 155, 115-131.

Lépine, Valérie, et De la Broise, Patrice (2012). Activités des commissions Formation - Recherche - Relations professionnelles. Revue française des sciences de l'information et de la communication, 1, s.p. (en ligne à : http://rfsic.revues.org/195).

Loneux, Catherine (2004). Savoirs professionnels et savoirs scientifiques dans l'organisation. Du face-à-face à l'enchevêtrement. Communication et langages, 141, 71-82.

Lutinier, Bruno, Dietsch, Bruno, et Sotto, Marie-Françoise (2011). Formations artistiques, culturelles et en communication en 2009: 155000 étudiants dans des filières très diversifiées. Culture chiffres, 5, 1-4.

Mercier, Arnaud (2007). Sciences de la communication et journalisme: de la compréhension des dérives à l'amélioration des pratiques. Studies in communication sciences, 7(1), 53-74.

Meyer, P. (1996). Why journalism needs Ph.D.s. The American Editor. September 1996, 10-11.

Miège. Bernard (1985). La Pensée communicationnelle. Grenoble: Presses universitaires de Grenoble.

Mœglin, Pierre (2005). Outils et médias éducatifs - Une approche communicationnelle. Grenoble : PUG.

Molière (1835). Euvres de Molière, précédées d'une notice sur sa vie et ses ouvrages par M. Sainte Beuve (Tome 1). Paris : Paulin.

Olsen, Richard, Weber, David et Trimble, Frank (2002). Cornerstones and Capstones: A Case Study on the Value of a Holistic Core in the Discipline of Communication Studies. Communication Education, 51(1), 65-80. 
Platon (1922). Phèdre ou De la beauté des âmes, traduit par Mario Meunier. Paris : Payot.

Radloff, Alex, de la Harpe, Barbara, et Zadnik, Marjan (1996). Developing science students' communication skills in the context of their discipline study: why we should and how we can. Actes de la Fourth International Conference on the Public Communication of Science and Technology. Barcelone: PCST Academy.

Sacré, Isabelle (1998). Communication et formation : programmes et réception. Communication et organisation, 14, 2-12.

Stephens, Mitchell (2010). Un manifeste pour l'enseignement du journalisme. Les Cahiers du journalisme, $21: 38-44$.

Winkin, Yves (1996). Anthropologie de la communication. Bruxelles: De Boeck.

\section{Références du corpus (ouvrages cités dans le texte uniquement)}

[ANT95J] Antoine, F., \& al. (1995). Ecrire au quotidien. Lyon : Chronique sociale.

[BLU88J] Blundell, W. E. (1988). The art and craft of feature writing. New York : Plume.

[CAP92J] Capon, R. J. (1991). Associated Press guide to news writing. New York : Macmillan.

[COL92G] Collectif (1992). Bien écrire et parler juste. Paris : Sélection du Reader's Digest.

[FLO84J] Florio, R. (1984). L'écriture de presse. Lille : Trimédia / ESJ.

[GER95J] Gergely, T. (1995). Ecrire ; information et persuasion. Bruxelles : De Boeck.

[GIR97G] Girard, B. (1997). La communication écrite dans l'entreprise. Bruxelles : De Boeck.

[GRI92G] Griselin, M., \& al. (1992). Guide de la communication écrite. Paris : Dunod.

[HER79J] Hervouet, L. (1979). Ecrire pour son lecteur. Lille: Trimédia / ESJ.

[LAY97G] Le Lay, Y. (1997). Savoir rédiger. Paris : Larousse. 
[LAZ93S] Laszlo, P. (1993). La vulgarisation scientifique. Paris: Presses universitaires de France.

[RIC92G] Richaudeau, F. (1995). Ecrire avec efficacité. Paris : Albin Michel.

[ROS94J] Ross, L. (1994). L'écriture de presse : l'art d'informer. Québec : Gaëtan Morin 